\title{
ERRATUM TO "ASYMPTOTIC DIMENSION AND BOUNDARY DIMENSION OF PROPER CAT(0) SPACES"
}

\author{
By \\ Naotsugu ChIneN and Tetsuya Hosaka
}

\begin{abstract}
The review on [1] in Mathematical Reviews points out that the proof of its main result is incorrect. The aim of this paper is to correct the previous paper's argument and clarify the statement.
\end{abstract}

In [2] it is stated that the proof of [1, Theorem 1.1] is incorrect, i.e., the map $f$ does not satisfy $(*)_{\rho}$, as claimed on line 4 of the first paragraph on [1, p. 188]. In fact, diam $f\left(B\left(\psi^{i_{k}}\left(x_{0}\right), 1\right)\right)=\operatorname{diam} a_{1}\left(B\left(x_{0}, 1\right)\right) \neq 0$ for each $k \in \mathbf{N}$. In this paper, we redefine the map $f=\bigcup_{k \in \mathbf{N}} f_{k}:(Y, \rho) \rightarrow\left(\mathbf{B}^{n+1}, \sigma\right)$, in particular $f_{k}: \psi^{i_{k}}\left(B\left(x_{0}, k\right)\right) \rightarrow \mathbf{B}^{n+1}$, where let $B\left(x_{0}, r\right)=\left\{y \in X: d\left(x_{0}, y\right) \leq r\right\}$ for $r>0$.

Let $(X, d)$ be a proper $\operatorname{CAT}(0)$ space and let $\psi:(X, d) \rightarrow(X, d)$ be an isometry satisfying that $\left\{\psi^{i}(x): i \in \mathbf{Z}\right\}$ is unbounded (see [1, Theorem 1.1]). Fix a point $x_{0}$ of $X$. For every $x \in X$, let $\xi_{x}:\left[0, d\left(x_{0}, x\right)\right] \rightarrow X$ be the geodesic from $x_{0}$ to $x$ in $(X, d)$. Recall the projection map $p_{1}: X \rightarrow B\left(x_{0}, 1\right)$ in [1, p. 187] defined by $p_{1}(x)=\xi_{x}\left(\min \left\{d\left(x_{0}, x\right), 1\right\}\right)$ for each $x \in X$.

Since $\left\{\psi^{i}(x): i \in \mathbf{Z}\right\}$ is unbounded, we have a sequence $i_{1}, i_{2}, \ldots$ of $\mathbf{N}$ satisfying an additional condition: $d\left(\psi^{i_{k}}\left(B\left(x_{0}, k\right)\right), \psi^{i_{k^{\prime}}}\left(B\left(x_{0}, k^{\prime}\right)\right)>\max \left\{k, k^{\prime}\right\}\right.$ whenever $k \neq k^{\prime}$ (see the second line from the bottom of [1, p. 187]). For every $k \in \mathbf{N}$, now we define a continuous map $q_{k}: B\left(x_{0}, k\right) \rightarrow B\left(x_{0}, 1\right)$ by $q_{k}(x)=$ $\xi_{x}\left(d\left(x_{0}, x\right) / k\right)$ for each $x \in B\left(x_{0}, k\right)$. Here, we redefine the map $f_{k}$ in the first line of $\left[1\right.$, p. 188] by a map $a_{1} \circ q_{k} \circ \psi^{-i_{k}}: \psi^{i_{k}}\left(B\left(x_{0}, k\right)\right) \rightarrow \mathbf{B}^{n+1}$. Let $Y=$ $\bigcup_{k \in \mathbf{N}} \psi^{i_{k}}\left(B\left(x_{0}, k\right)\right)$ and let $f=\bigcup_{k \in \mathbf{N}} f_{k}: Y \rightarrow \mathbf{B}^{n+1}$.

We see that $\left.p_{1}\right|_{B\left(x_{0}, k\right)}$ is homotopic to $q_{k}$. Indeed, we have a homotopy $H: B\left(x_{0}, k\right) \times[0,1] \rightarrow B\left(x_{0}, 1\right):\left.p_{1}\right|_{B\left(x_{0}, k\right)} \simeq q_{k}$ defined by

$$
H(x, t)=\xi_{x}\left(\left(d\left(x_{0}, x\right) / k-\min \left\{d\left(x_{0}, x\right), 1\right\}\right) t+\min \left\{d\left(x_{0}, x\right), 1\right\}\right)
$$

2000 Mathematics Subject Classification: Primary 20F69; Secondary 20F65, 54F45.

Key words and phrases: asymptotic dimension; CAT(0) space; Higson corona; boundary. Received July 23, 2014. 
for each $(x, t) \in B\left(x_{0}, k\right) \times[0,1]$. Thus, $\quad 0 \neq\left[a_{k}\right]=\left[a_{1} \circ p_{1}\right]=\left[a_{1} \circ q_{k}\right] \in$ $H^{n+1}\left(B\left(x_{0}, k\right), S\left(x_{0}, k\right)\right)$, where $a_{k}:\left(B\left(x_{0}, k\right), S\left(x_{0}, k\right)\right) \rightarrow\left(\mathbf{B}^{n+1}, \mathbf{S}^{n}\right)$ is the map in the fifth line from the bottom of [1, p. 187]. Therefore, every $f_{k}$ is essential.

We show that $f:(Y, \rho) \rightarrow\left(\mathbf{B}^{n+1}, \sigma\right)$ has property $(*)_{\rho}$ : for every $r>0$ and every $\varepsilon>0$, there exists a compact set $K$ of $Y$ such that diam $f(B(x, r) \cap Y)<\varepsilon$ for all $x \in Y \backslash K$. Here $\rho=\left.d\right|_{Y}$ and $\sigma$ is the usual metric of $\mathbf{B}^{n+1}$. Let $r>0$ and let $\varepsilon>0$. By the uniformly continuity of $a_{1}$, there exists $\delta>0$ such that for every $E \subset B\left(x_{0}, 1\right)$ with $\operatorname{diam} E<\delta$, $\operatorname{diam} a_{1}(E)<\varepsilon$. We see that for every $r>0$ there exists $k_{0} \in \mathbf{N}$ with $k_{0}>\max \{r, 4 r / \delta\}$ such that for every $k \geq k_{0}$ and every $D \subset B\left(x_{0}, k\right)$ with $\operatorname{diam} D \leq 2 r$, diam $q_{k}(D)<\delta$. Let $k \geq k_{0}$ and let $x, y \in B\left(x_{0}, k\right)$ with $d(x, y) \leq 2 r$. We note that $q_{k}(x)=\xi_{x}\left(d\left(x_{0}, x\right) / k\right)$ and $q_{k}(y)=\xi_{y}\left(d\left(x_{0}, y\right) / k\right)$. By the comparison triangle for $\triangle\left(x_{0}, x, y\right)$, we have $d\left(q_{k}(x), q_{k}(y)\right) \leq d(x, y) / k \leq 2 r / k<\delta / 2$. Thus, every $D \subset B\left(x_{0}, k\right)$ with $\operatorname{diam} D$ $\leq 2 r$ satisfies that diam $q_{k}(D)<\delta$. Let $K=\bigcup_{k=1}^{k_{0}-1} \psi^{i_{k}}\left(B\left(x_{0}, k\right)\right)$, let $x \in Y \backslash K$ and let $k \in \mathbf{N}$ with $k \geq k_{0}$ such that $x \in \psi^{i_{k}}\left(B\left(x_{0}, k\right)\right)$. Since $B(x, r) \cap Y \subset \psi^{i_{k}}\left(B\left(x_{0}, k\right)\right)$, $\psi^{-i_{k}}(B(x, r) \cap Y)=B\left(\psi^{-i_{k}}(x), r\right) \cap B\left(x_{0}, k\right) \subset B\left(x_{0}, k\right)$. Since $\operatorname{diam} \psi^{-i_{k}}(B(x, r)$ $\cap Y) \leq 2 r$, by the above we see that $\operatorname{diam} q_{k} \circ \psi^{-i_{k}}(B(x, r) \cap Y)<\delta$. Hence $\operatorname{diam} f(B(x, r) \cap Y)=\operatorname{diam} f_{k}(B(x, r) \cap Y)=\operatorname{diam} a_{1}\left(q_{k} \circ \psi^{-i_{k}}(B(x, r) \cap Y)\right)<\varepsilon$. Therefore, the map $f$ has property $(*)_{\rho}$.

Thus, there exists an extension $\bar{f}: \bar{Y}^{\rho} \rightarrow \mathbf{B}^{n+1}$ of $f$. By the same manner on the second paragraph of [1, p. 188], we can show that $g=\left.\bar{f}\right|_{v_{\rho} Y}: v_{\rho} Y \rightarrow \mathbf{B}^{n+1}$ is essential because every $f_{k}$ is essential. Therefore, $\operatorname{dim} v_{d} X \geq \operatorname{dim} v_{\rho} Y \geq n+1$.

\section{References}

[ 1 ] N. Chinen and T. Hosaka, Asymptotic dimension and boundary dimension of proper CAT $(0)$ spaces, Tsukuba J. Math. 36 (2012), 185-191.

[2] X. Xie, The Mathematical Review on "Asymptotic dimension and boundary dimension of proper CAT(0) spaces, Tsukuba J. Math. 36 (2012), 185-191”.

Department of Mathematics

National Defense Academy of Japan

Yokosuka 239-8686, Japan

E-mail: naochin@nda.ac.jp

Department of Mathematics

Shizuoka University, Suruga-ku

Shizuoka 422-8529, Japan

E-mail: sthosak@ipc.shizuoka.ac.jp 